\title{
Planning Active Cannula Configurations Through Tubular Anatomy
}

\author{
Lisa A. Lyons, Robert J. Webster III, and Ron Alterovitz
}

\begin{abstract}
Medical procedures such as lung biopsy and brachytherapy require maneuvering through tubular structures such as the trachea and bronchi to reach clinical targets. We introduce a new method to plan configurations for active cannulas, medical devices composed of thin, pre-curved, telescoping lumens that are capable of following controlled, curved paths through open or liquid-filled cavities. Planning optimal configurations for these devices is challenging due to their complex kinematics, which involve both beam mechanics and space curves. In this paper, we propose an optimization-based planning algorithm that computes active cannula configurations through tubular structures that reach specified targets. Given the target location, the start position and orientation, and a geometric representation of the physical environment extracted from pre-procedure medical images, the planner optimizes insertion length and orientation angle of each lumen of the active cannula. The planner models active cannula kinematics using a physically-based simulation that incorporates beam mechanics and minimizes energy. The algorithm typically computes plans in less than 2 minutes on a standard PC. We apply the method in simulation to anatomy extracted from a human CT scan and demonstrate configurations for a 5-lumen active cannula that maneuver it through the bronchi to targets in the lung.
\end{abstract}

\section{INTRODUCTION}

Lung cancer is the leading cause of cancer-related death in the United States with over 150,000 deaths per year [5]. Diagnosing and treating lung cancer at its earlier stages could significantly improve patient outcomes; the 5-year survival rate for patients diagnosed with early stage cancer is more than 5 times greater than for those with late stage diagnoses [16], [29]. However, many sites within the lung, such as peripheral nodules identified in CT scans, are difficult or impossible to access for biopsy and treatment delivery using currently available tools. Current tools either cannot access sites deep within the lung or carry a significant risk of complications such as pneumothorax (collapse of the lung). This makes definitive early diagnosis and treatment impossible for many patients. In this paper, we explore planning configurations for active cannulas, devices composed of thin, pre-curved, telescoping lumens (tubes) that have the potential to safely maneuver through the bronchi of the lung to clinical targets for biopsy or brachytherapy treatment, as shown in Fig. 1.

Active cannulas are needle-like devices that can be controlled to travel in curved trajectories through open or liquidfilled cavities as well as soft tissues. Each lumen, composed

L. A. Lyons and R. Alterovitz are with the Department of Computer Science, University of North Carolina at Chapel Hill, USA llyons@email.unc.edu, ron@cs.unc.edu

R. J. Webster is with the Department of Mechanical Engineering, Vanderbilt University, Nashville, TN, USA robert.webster@vanderbilt.edu

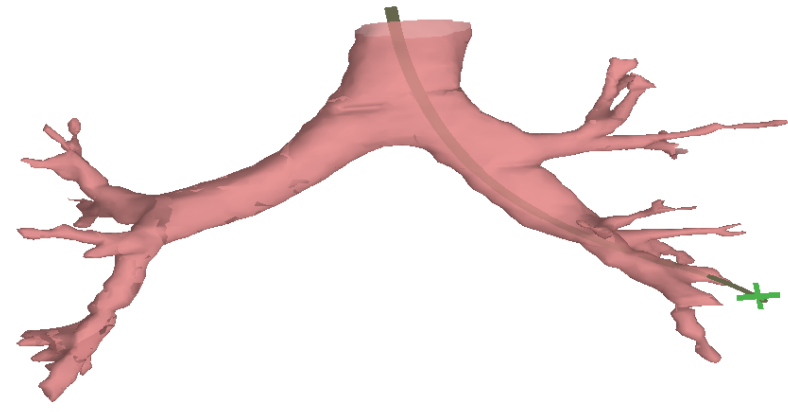

Fig. 1. Illustration of an active cannula composed of 5 concentric lumens maneuvering through the bronchial tubes to reach a target (green cross). The bronchial tube model was extracted from a human CT image [23]. The active cannula can be brought to the start location using standard bronchoscopes. The active cannula configuration reaching the target was computed by our planner.

of nitinol, is comprised of a long, straight transmission section followed by a pre-curved section prior to the tip [13], [26], [33]. Each lumen has two degrees of freedom; it may be twisted axially or extended outward with respect to its containing lumen. The movement of the lumens may be controlled by either humans or robotic actuators. The wide range of configurations offered by these devices makes them ideal for minimally invasive surgical procedures where a straight needle cannot be used because of the potential damage to the surrounding tissue. Potential applications of active cannulas include reaching targets in the lung via the throat, maneuvering through the brain for biopsy or electrode implantation [20], and accessing sites in the fetus within the womb [14].

Planning lumen configurations (translations and rotations applied at lumen bases) that correspond to desired spatial tip coordinates or shaft curves is not intuitive, motivating the need for efficient planning algorithms. For planning problems in tubular environments like the lung, the inputs to planning are a start pose, a target location, and a network of tubular pathways extracted from medical images. We present an optimization based planner that (1) uses physically-based simulation to accurately model active cannula shape as a function of configuration inputs, and (2) optimizes configuration inputs to ensure the active cannula reaches a target while minimizing distance traveled outside tubular pathways. This planner introduces two key extensions to our prior work [19] on motion planning for active cannulas: (1) we compute active cannula shape by minimizing energy to capture the interaction between lumens, and (2) we consider motion through tubular structures rather than avoidance of spherical obstacles. 


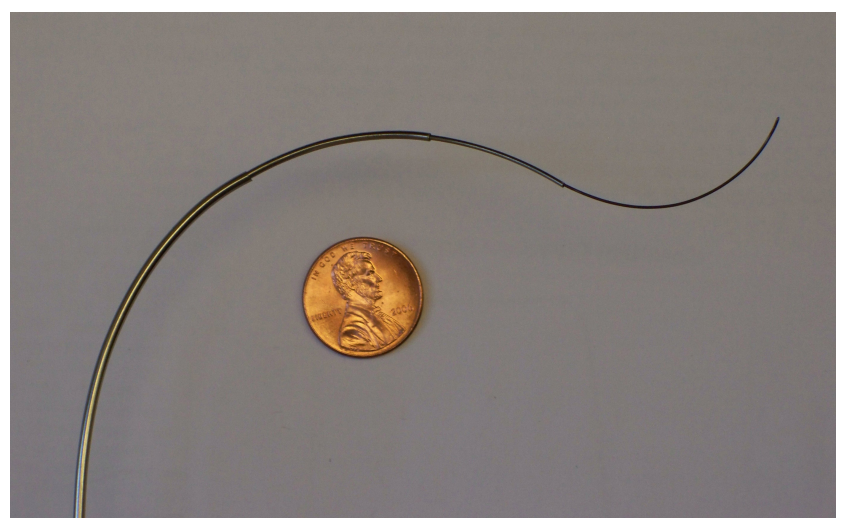

Fig. 2. An active cannula with 4 concentric lumens. Each concentric lumen is pre-shaped with a different radius of curvature, with the thinner, inner lumens having higher curvature.

We apply the planner to active cannulas composed of fully pre-curved lumens as well as those composed of lumens with straight transmission sections followed by curved sections. For the former, the configurations computed by our planner can be directly transformed into motion plans under certain model assumptions by appropriately sequencing lumen deployment. For the latter, motion plans may be executed but are not guaranteed to be feasible due to the time-dependent shape changes that occur during lumen deployment. We apply our planner to anatomical test cases based on lung procedures where we extract tubular structures (the bronchi) from a human CT volumetric image. We compute viable configurations for active cannulas that maneuver through the bronchi to reach targets not directly accessible by straight line paths.

\section{Related Work}

Optimization-based motion planning has been used to plan minimally invasive surgical procedures involving needle insertion. The aim of these planners is to compute an optimal configuration for the robot before motion begins based on an objective function. Optimization techniques have been used to account for tissue deformation as a result of needle insertion for stiff, symmetric-tip needles in 2D [3] and in 3D [10]. Alterovitz et al. applied this approach to maneuver flexible, bevel-tip steerable needles around obstacles in soft tissues [2]. DiMaio et al. [11] and Glozman et al. [15] have explored simulation and planning for flexible symmetrictip needles in 2D deformable tissue. Their approach is to translate and rotate the needle base perpendicular to the insertion direction while inserting the needle, relying on tissue deformations to maneuver around obstacles.

Optimization-based motion planning builds on the idea behind potential fields, virtual fields that robots can follow to reach a target position while avoiding obstacles [8], [17]. They are frequently used as vector fields where one can find the optimal velocity vector at any point in space [9], [17], [21]. In this work, we do not compute a vector field but rather find a locally optimal configuration for an active cannula given a target and a cost function.
Like active cannulas, steerable needles follow curved paths, although only through soft tissues. Optimizationbased motion planning has been applied to steerable needles inserted in 2D tissue slices around polygonal obstacles [2]. Other approaches include diffusion-based motion planning to numerically compute a path in 3D stiff tissues [24], screwbased motion planning to compute steerable needle paths in 3D around spherical obstacles [12], rapidly exploring random trees [34], and planning methods that explicitly consider uncertainty in the needle's motion to maximize the probability of successfully reaching the target [1], [4]. The latter method, combined with an imaging and control system, has been successfully integrated with robot hardware [25]. Though these concepts serve as a guide for motion planning for active cannulas, the methods cannot be directly applied here because active cannulas are fundamentally physically and mechanically different from steerable needles.

In terms of mechanical design, active cannulas can be considered a generalization of catheter and needle steering strategies. The key difference between active cannulas and single-element designs like catheters or steerable needles is that an active cannula need not rely completely on tissue reaction forces to steer. Rather, it can change shape using internal reaction moments that tubes apply to one another. Thus, active cannulas are properly considered miniature robotic manipulators, and belong to the continuum robot family due to their continuously flexible backbone [26], [33]. The most complete available model of active cannula shape accounts for tube interaction using beam mechanics. It captures the effects of bending, torsion, and variable precurvature in an arbitrary number of tubes (see [26] and [13]), yielding the shape of the active cannula as a function of tube base translation positions and axial angles. It is the most descriptive and accurate in a series of models [14], [26]-[28], [32], [33] derived in the past few years, and includes all prior models as special cases. One of these special cases assumes infinite torsional rigidity beyond the straight transmission of the lumens [33]. This model constitutes the active cannula as a set of "links." Each link has a unique curvature and axial angle with respect to the previous link. These curvatures and axial angles can be computed by performing an energy minimization. This is the model we use in this paper.

The relationship between active cannula control inputs (translations and rotations applied at lumen bases) and the desired spatial tip coordinates or shaft curves is not intuitive to humans. Algorithms that compute active cannula configurations subject to constraints can address this challenge. Active cannulas with a sufficient number of lumens are a type of redundant manipulator. Recent work has explored probabilistic planning algorithms for holonomic redundant manipulators [7], [31], but work that considers planning subject to the kinematic constraints of active cannulas is more limited. This work extends our previous work in motion planning for active cannulas [19], which considered avoidance of spherical obstacles and used a simplified kinematic model of active cannulas that neglected beam mechanics and lumen interactions. In this paper, we utilize a better model 


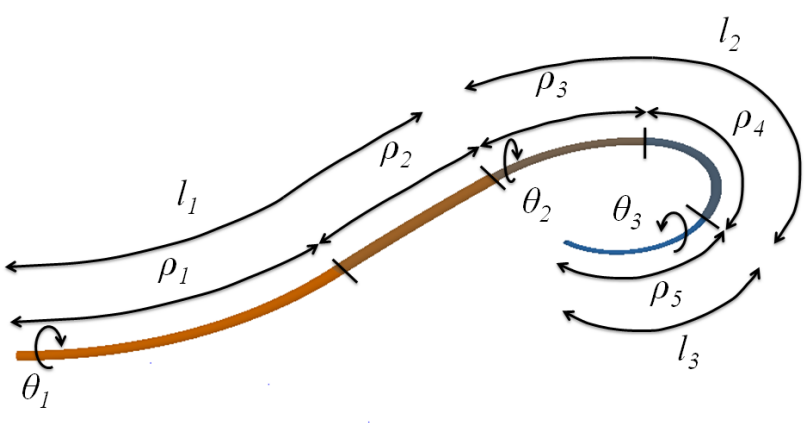

Fig. 3. A cannula with $N=3$ lumens has $S=5$ links with configuration $\mathbf{q}=\left(l_{1}, \theta_{1}, l_{2}, \theta_{2}, l_{3}, \theta_{3}\right)$. In this configuration, the curved portion of the second lumen begins where $\rho_{1}$ and $\rho_{2}$ meet and ends where $\rho_{4}$ and $\rho_{5}$ meet. Therefore, $d_{2}$ spans $\rho_{3}, \rho_{4}$, and $\rho_{5}$.

of active cannula shape that models lumen interactions and requires an energy minimization during every iteration of the planning method. We also consider tubular environments, enabling the application of the planner to medical procedures in the lung.

\section{Problem Formulation}

First, we will define the physical model for the active cannula that we use. We then define tubular environments (e.g. bronchial tubes extracted from medical images). Finally, we will describe the problem of computing configurations for active cannulas through tubular environments.

\section{A. Physical Model}

Our model of active cannulas is based on the beam mechanics formulation presented by Webster et al. [33]. We approximate the active cannula as moving through open air and do not consider the physical forces acting on the cannula due to interactions with tissues. We consider an active cannula with $N$ lumens numbered in order of decreasing diameter. Each lumen $i$ consists of a straight transmission of length $L_{i}$, followed by a pre-curved portion of length $d_{i}$. Each lumen also has bending stiffness $E_{i} I_{i}$ where $E_{i}$ is the modulus of elasticity and $I_{i}$ is the cross-sectional moment of inertia for lumen $i$. The pre-curved portions have resting radii of curvature given by $r_{i}, i=1, \ldots, N$. This model assumes that the cannula is infinitely torsionally rigid beyond the straight transmission.

When sections of two or more lumens overlap at some point, the resulting curvature at that point may be different from the rest curvature of any lumen at that point due to interactions between the lumens. Based on the active cannula model [33], we divide the active cannula into segments called links where the curvature $\kappa_{j}$ and axial axial angle $\phi_{j}$ (known as the arc parameters) are constant. An active cannula with $N$ lumens has $M=2 N-1$ links whose length we denote by $\rho_{j}, j=1, \ldots, M$, as shown in Fig. 3 .

Each lumen has two degrees of freedom; it may be (1) extended or retracted from the previous lumen and (2) axially rotated, as shown in Fig. 3. We define the configuration for an active cannula as the $2 N$ dimensional vector $\mathbf{q}=\left(l_{i}, \theta_{i}\right.$ : $i=1, \ldots, N)$ where $l_{i}$ is the translated length of the $i$ 'th lumen and $\theta_{i}$ is the axial angle at the base of the $i$ 'th lumen. We assume that the active cannula is inserted at a given point in $3 \mathrm{D}$ space $\mathbf{x}_{\text {start }}$ and is oriented along the vector $\mathbf{v}_{\text {start }}$.

We use an energy minimization to determine the arc parameters from the configuration as in [33]. The objective is to express the shape of the needle (a space curve) as a function of the input translations and rotations applied at the base of each component lumen. As a result of axial moments generated during twisting, 'wind-up' of the lumens occurs. As a consequence, a link's axial angle $\phi$ may be different from the angle $\theta$ at the base of the corresponding lumen.

We define the function $f(\mathbf{q}): \mathbb{R}^{2 N} \mapsto \mathbb{R}^{3}$ as returning the tip position in $3 \mathrm{D}$ space given the active cannula configuration q. Since each link has its own unique curvature $\kappa_{j}$ and axial angle $\phi_{j}$, there is a unique transformation $T_{j}\left(\kappa_{j}, \phi_{j}\right)$ that transforms the start coordinate of a link to the endpoint of that link. So, we define

$$
f(\mathbf{q})=T_{m} T_{m-1} \cdots T_{1} \mathbf{x}_{\text {start }} .
$$

\section{B. Tubular Environments}

We apply our planning algorithm to problems involving tubular environments in which the active cannula should remain inside a tube and damage the least amount of sensitive tissue outside the tube. As a motivating example, we focus on lung biopsy procedures. During a lung biopsy, the physician would like to guide a device through the bronchial tubes as long as possible to incur the least tissue damage en route to a nodule, which may or may not be inside a bronchial tube. To evaluate the potential of our planner, we used bronchial tubes extracted from a human CT scan (the TRAGICOMIX dataset [23]).

From the CT scan, we extracted the bronchial tube structure and represented it using a 3D triangular surface mesh model. The bronchial tree mesh was extracted using the OsiriX Region of Interest (ROI) Growing Tool [23]. Within this model, we manually define a "skeleton" consisting of a series of connected vertices. These vertices are defined as a set $P$ of size $L, P=\left\{P_{1}, P_{2}, \ldots, P_{L}\right\}$. Each vertex also has a corresponding radius based on the radius of the tube at that point. We treat the bronchial tree as a rigid body, and leave consideration of bronchial tissue deformations to future work.

\section{Planning Problem}

The planning problem consists of two parts, minimizing the Euclidean distance from the tip of the cannula to the target $\mathbf{x}_{\text {target }}$ and keeping the cannula inside the tube structure en route to the target. The start location for the active cannula in 3D space is $\mathbf{x}_{\text {start }}$.

Given the active cannula properties, the start configuration, the target coordinate, and the bronchial tree structure, we formulate the motion planning problem as a nonlinear, constrained optimization problem. First, we require that the active cannula tip reach the target, which we represent as the constraint:

$$
\left\|f(\mathbf{q})-\mathbf{x}_{\text {target }}\right\|=0 .
$$



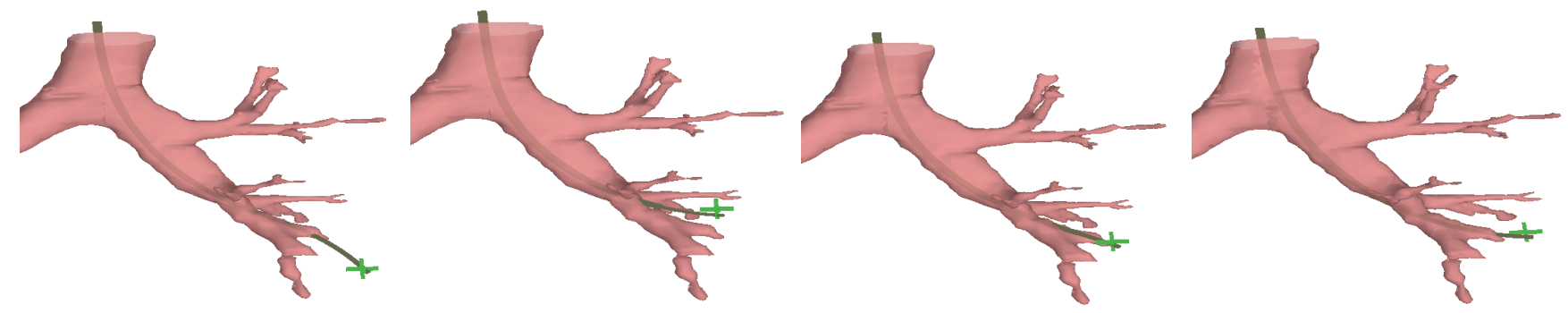

Fig. 4. Four active cannula configurations computed by the planner that reach the target (green cross) and optimally satisfy the cost metric. The images are cropped to enhance the relevant portion of the anatomy.

We also prefer solutions that minimize the total cost function $c(\mathbf{q})$. This function defines what (if any) penalty is incurred when the active cannula moves outside the tubular structure. We represent this cost mathematically by integrating the values of a potential field along the cannula.

$$
c(\mathbf{q})=\int_{S} g(\mathbf{s}) d \mathbf{s}
$$

where $\mathbf{s}$ is a point along the curve $S$ describing the cannula and $g(\mathbf{s}): \mathbb{R}^{3} \mapsto \mathbb{R}^{1}$ is a cost function, which we will define for our problem in Section IV. Finally, we require that $l_{i} \geq 0$, $i=1, \ldots, N$ as required by the active cannula model.

The motion planning problem may now be formulated as a nonlinear, constrained optimization problem:

$$
\begin{aligned}
& \mathbf{q}^{*}=\underset{\mathbf{q}}{\operatorname{argmin}} c(\mathbf{q}) \\
& \text { Subject to: } \\
& \quad\left\|f(\mathbf{q})-\mathbf{x}_{\text {target }}\right\|=0 \quad \\
& \quad l_{i} \geq 0
\end{aligned}
$$

where $\mathbf{q}^{*}$ is the optimal active cannula configuration.

The active cannula configuration computed using this approach also serves as a motion plan under certain assumptions. For active cannulas composed of lumens that are fully curved and have no straight transmission segments, we can achieve the computed optimal configuration by first axially rotating each lumen to its respective angle and then sequentially deploying each link by simultaneously deploying the link's constituent lumens. Under the model of active cannulas we use in this paper, this insertion sequence ensures that the deployment of later links will not affect the shape of those already deployed. In the more general case of active cannulas with straight transmissions, the lumens deform and twist each other such that deployment of any lumen will in general affect the shape of already deployed lumens. Computing a plan for such active cannulas requires considering at the planning stage the time-dependent process of deploying the lumens. In future work, we will address these cases by exploring the use of milestones along the skeleton or inside the tubes to compute sequences of configurations that minimize costs.

\section{Planning Algorithm}

The optimization formulation in equation 3 minimizes the cost function $c(\mathbf{q})$ while requiring that the active cannula tip reach the target (i.e. satisfy equation 1 ). This requires solving one optimization problem (compute the active cannula shape by minimizing energy) inside another optimization problem (compute the translation and axial angle of each tube to solve the planning problem). To compute active cannula shape, we minimize energy using the L-BFGS algorithm [18].

To solve the planning problem, we compute a locally optimal configuration $\mathbf{q}^{*}$ using a penalty method. Penalty methods solve a constrained optimization problem by converting it to a series of unconstrained optimization problems [6]. For convex nonlinear problems, the method will generate points that converge arbitrarily close to the global optimal solution [6]. For nonconvex problems, the method can only converge to a local optimal solution.

Applying the penalty method, we move the constraint that the active cannula reaches the target $\left\|f(\mathbf{q})-\mathbf{x}_{\text {target }}\right\|=0$ to the objective function and define a new optimization problem:

$$
\begin{aligned}
& \mathbf{q}^{*}=\underset{\mathbf{q}}{\operatorname{argmin}}\left(c(\mathbf{q})+\mu\left\|f(q(\mathbf{u}))-\mathbf{x}_{\text {target }}\right\|\right) \\
& \text { Subject to: } \\
& \quad l_{i} \geq 0 \quad i=1, \ldots, N
\end{aligned}
$$

The remaining constraints are limit constraints that can easily be handled by unconstrained minimization algorithms.

We iteratively increase $\mu$ by a constant factor $\beta$ until either $\left\|f(\mathbf{q})-\mathbf{x}_{\text {target }}\right\| \leq \delta$ where $\delta$ is some small value or we reach a maximum $\mu^{\prime}$ which we define to maintain numerical stability. Decreasing $\delta$ results in a more accurate result but requires more computation time.

In order to calculate $c(\mathbf{q})$, we first discretize the cannula into a series of $A$ points where $A$ depends on the number of links $M$. Recall from equation 2 that the cost function is an integral over the curve of the entire cannula. So, we take the sum of the cost contributions from each of the points $\mathbf{x}_{a}$ to compute $c(\mathbf{q})$. We parameterize the skeleton along a particular tube by $s \in[0,1)$. For each point, $\mathbf{x}_{a}$, we find the corresponding point on the skeleton structure $\mathbf{x}_{\text {skeleton }}$ that is $s=a / A$ along the skeleton. The cost contribution for $\mathbf{x}_{a}$ is dependent on its shortest distance to the skeleton and whether it is within the bronchial tube. We define the cost function for a point as

$$
g\left(\mathbf{x}_{a}\right)=\left\{\begin{aligned}
d^{2} & \text { if } d \leq r \\
(d-r)^{3}+r^{2} & \text { if } d>r
\end{aligned}\right.
$$




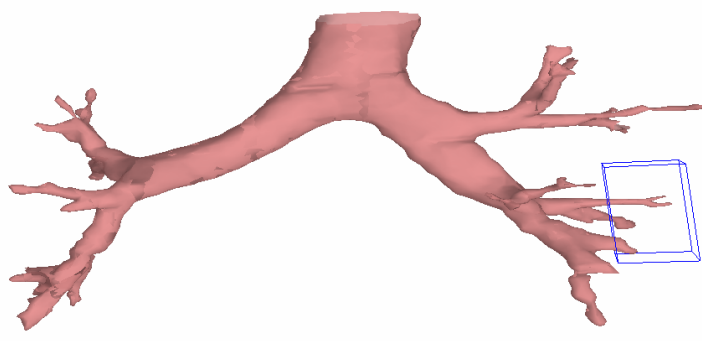

Fig. 5. We evaluated the planner by computing configurations to reach targets uniformly randomly sampled inside the blue box.

where $r$ is the approximate radius of the bronchial tube at the point $x_{\text {skeleton }}$ and $d$ is the distance from $\mathbf{x}_{\text {skeleton }}$ to $\mathbf{x}_{a}$. We define the total cost function for an active cannula configuration $\mathbf{q}$ as $c(\mathbf{q})=\sum_{A} g\left(\mathbf{x}_{a}\right)$.

We use the L-BFGS algorithm to find a locally optimal solution to 4 for a given starting plan $\mathbf{q}_{0}$ [18], [22]. For each gradient direction, we use Armijo's Rule [6] for line search with an initial movement distance of $\epsilon=0.0001$.

Since $\mathbf{q}^{*}$ is a locally optimal solution, there may exist a better global optimum. To improve the quality of computed solutions, we select 10 random, uniformly distributed initial values for $\mathbf{q}_{0}$ and execute the local optimization for each. The initial values were chosen using the following criteria for the lumens: $0 \leq l_{i}<r_{i}$ and $0 \leq \theta_{i}<2 \pi \forall i$. Although we automatically select the optimal solution based on the lowest value of $c(\mathbf{q})$, physicians or engineers may also choose among the solutions found to optimize other criteria, such as shortest lumen deployment lengths, minimum torsion, more rapidly healing tissues, etc.

\section{Results}

The results of the planner are illustrated for the lung scenario described earlier and shown in Fig. 1 and Fig. 4 for a cannula with $N=5$ lumens ( $M=9$ links). The visualizations were generated using OpenGL and the model of the human bronchi was extracted from a CT scan as described in Sec. III-B.

In order to evaluate the planner, we ran the method for 100 targets randomly selected from within the box in Fig. 5. We used active cannulas with $N=5$ concentric lumens with radii of curvature of $r_{1}=7 \mathrm{~cm}, r_{2}=5 \mathrm{~cm}, r_{3}=5 \mathrm{~cm}$, $r_{4}=4 \mathrm{~cm}$, and $r_{5}=3 \mathrm{~cm}$. Their curved segment lengths were $d_{1}=7 \mathrm{~cm}, d_{2}=6 \mathrm{~cm}, d_{3}=6 \mathrm{~cm}, d_{4}=4 \mathrm{~cm}$, and $d_{5}=4 \mathrm{~cm}$. We also considered active cannulas with the same properties except that they only contained curved segments. The workspace was approximately $20 \mathrm{~cm}$ in all directions. To reduce the effects of local minima, for each target we solve the optimization for each of the ten randomly chosen initial conditions and select the lowest cost solution. Each of these plans was computed in under 2 minutes on a 3.33 $\mathrm{GHz}$ Intel@ i7-975 PC with 8 GB RAM.

For these experiments, the Euclidean distance between the tip of the cannula and the target must be less than $\delta=0.07$ $\mathrm{cm}$ to satisfy the target constraint. To compute the cost function, we use $S=5 M=45$ short segments to represent

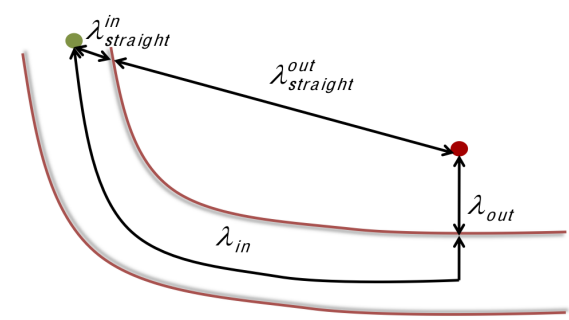

Fig. 6. We evaluate active cannula plans by comparing the configurations to an ideal configuration (shown as $\lambda_{\text {in }}$ through $\lambda_{\text {out }}$ ) where the device remains inside the bronchial tube and then takes a right angle turn to the target, minimizing damage to sensitive tissues outside the tube. We also compare to a straight needle path (shown as $\lambda_{\text {straight }}$ ), which must cut through substantially more sensitive tissues en route to the target.

TABLE I

COMPUTATION TIMES AND QUALITY METRICS FOR 100 TRIALS IN THE ENVIRONMENT SHOwn in Fig. 5. All tests were RUN on a 3.33 GHz INTEl@ I7-975 PC With 8 GB RAM.

\begin{tabular}{|r|r|r|r|r|r|}
\hline & Time (sec) & $\frac{S_{\text {out }}}{\lambda_{\text {out }}}$ & $\frac{S_{\text {in }}}{\lambda_{\text {in }}}$ & $\frac{S_{\text {out }}}{\lambda_{\text {straight }}^{\text {out }}}$ & $\frac{S_{\text {in }}}{\lambda_{\text {straight }}^{\text {in }}}$ \\
\hline Mean & 82.0 & 3.28 & 0.741 & 0.456 & 3.36 \\
Median & 77.9 & 2.30 & 0.807 & 0.365 & 3.53 \\
$\sigma$ & 20.2 & 2.28 & 0.176 & 0.223 & 0.984 \\
\hline
\end{tabular}

the cannula. For numerical stability, $\mu$ was only allowed to increase by a maximum factor of $10^{8}$ from its initial value.

We achieved a $100 \%$ success rate for reaching the target, both for active cannulas with curved and straight sections and for active cannulas with only curved sections. Table I shows the mean, median, standard deviation for the computation time to produce plans for environments corresponding to those shown in Fig. 5 involving active cannulas with both straight and curved sections. Ideally, the cannula would be able to remain inside the bronchi as far as possible and make a right angle turn directly toward the target for the least tissue damage possible (see Fig. 6). In Table I, we compare our solutions to this ideal and to a straight needle beginning at $\mathbf{x}_{\text {start }}$ and ending at the target. The table shows the mean ratio of the distance the cannula was outside the bronchi $\left(S_{\text {out }}\right)$ to the ideal distance from the bronchi to the target $\left(\lambda_{\text {out }}\right)$ as well as the mean ratio of the distance the cannula spends inside the bronchi $\left(S_{\text {in }}\right)$ to the ideal distance $\left(\lambda_{\text {in }}\right)$. It also shows the same ratios for active cannulas compared to straight needles $\left(\lambda_{\text {straight }}^{\text {in }}\right.$ and $\left.\lambda_{\text {straight }}^{\text {out }}\right)$.

\section{Conclusion}

We introduced a new method to plan configurations for active cannulas through tubular structures. The planner optimizes the insertion length and axial angle of each lumen of the active cannula such that the device maneuvers through tubular structures to a target. The method requires as input the target position, the start position and orientation, the mechanical properties of the active cannula's components, and a geometric representation of the physical environment extracted from pre-procedure medical images. The planner then computes the active cannula configuration such that it reaches the target while minimizing a cost metric based on 
cutting tissues outside the tubes. The planner models active cannula kinematics using a physically-based simulation that incorporates beam mechanics and minimizes energy. The algorithm typically computes plans in less than 2 minutes on a standard PC. We applied the method in simulation to anatomy extracted from a human CT scan and demonstrated configurations for a 5-lumen active cannula that maneuver through the bronchi to targets in the lung.

In future work, we plan to take steps to bring motion planning for active cannulas closer to clinical application. We plan to automate the method for finding the skeletal structure of the bronchial tubes using a method akin to those currently used in segmentation and computer graphics (e.g. [30]). We also plan to develop a planner with full, timedependent motion planning capabilities and to integrate the planner into a robotic system with image guidance.

\section{ACKNOWLEDGEMENT}

This research was supported in part by the National Science Foundation (NSF) under grant \#IIS-0905344. The authors thank Dr. Richard Feins from Cardiothoracic Surgery at UNC-Chapel Hill for his feedback on the clinical application of active cannulas to lung procedures. The authors also thank Caleb Rucker for the photo in Fig. 2.

\section{REFERENCES}

[1] R. Alterovitz, M. Branicky, and K. Goldberg, "Motion planning under uncertainty for image-guided medical needle steering," Int. J. Robotics Research, vol. 27, no. 11-12, pp. 1361-1374, Nov. 2008.

[2] R. Alterovitz, K. Goldberg, and A. M. Okamura, "Planning for steerable bevel-tip needle insertion through 2D soft tissue with obstacles," in Proc. IEEE Int. Conf. Robotics and Automation (ICRA), Apr. 2005, pp. $1652-1657$.

[3] R. Alterovitz, J. Pouliot, R. Taschereau, I.-C. Hsu, and K. Goldberg, "Sensorless planning for medical needle insertion procedures," in Proc. IEEE/RSJ Int. Conf. on Intelligent Robots and Systems (IROS), Oct. 2003, pp. 3337-3343.

[4] R. Alterovitz, T. Siméon, and K. Goldberg, "The Stochastic Motion Roadmap: A sampling framework for planning with Markov motion uncertainty," in Robotics: Science and Systems III (Proc. RSS 2007), W. Burgard, O. Brock, and C. Stachniss, Eds. Cambridge, MA: MIT Press, 2008, pp. 246-253.

[5] American Cancer Society, "Cancer facts \& figures 2009," American Cancer Society, Tech. Rep., 2009.

[6] M. S. Bazaraa, H. D. Sherali, and C. M. Shetty, Nonlinear Programming: Theory and Algorithms, 2nd ed. New York, NY: John Wiley \& Sons, Inc., 1993.

[7] D. Bertram, J. Kuffner, R. Dillmann, and T. Asfour, "An integrated approach to inverse kinematics and path planning for redundant manipulators," in Proc. IEEE Int. Conf. Robotics and Automation (ICRA), May 2006, pp. 1874-1879.

[8] H. Choset, K. M. Lynch, S. Hutchinson, G. Kantor, W. Burgard, L. E. Kavraki, and S. Thrun, Principles of Robot Motion: Theory, Algorithms, and Implementations. MIT Press, 2005.

[9] C. I. Connolly, J. B. Burns, and R. Weiss, "Path planning using Laplace's equation," in Proc. IEEE Int. Conf. Robotics and Automation (ICRA), May 1990, pp. 2102-2106.

[10] E. Dehghan and S. E. Salcudean, "Needle insertion point and orientation optimization in non-linear tissue with application to brachytherapy," in Proc. IEEE Int. Conf. Robotics and Automation (ICRA), Apr. 2007, pp. 2267-2272.

[11] S. P. DiMaio and S. E. Salcudean, "Needle steering and motion planning in soft tissues," IEEE Trans. Biomedical Engineering, vol. 52, no. 6, pp. 965-974, Jun. 2005.

[12] V. Duindam, R. Alterovitz, S. Sastry, and K. Goldberg, "Screw-based motion planning for bevel-tip flexible needles in 3D environments with obstacles," in Proc. IEEE Int. Conf. Robotics and Automation (ICRA), May 2008, pp. 2483-2488.
[13] P. E. Dupont, J. Lock, and E. Butle, "Torsional kinematic model for concentric tube robots," in Proc. IEEE Int. Conf. Robotics and Automation (ICRA), May 2009, pp. 3851-3858.

[14] J. Furusho, T. Katsuragi, T. Kikuchi, T. Suzuki, H. Tanaka, Y. Chiba, and H. Horio, "Curved multi-tube systems for fetal blood sampling and treatments of organs like brain and breast," J. Comput. Assist. Radiol. Surg., vol. 1, pp. 223-226, 2006.

[15] D. Glozman and M. Shoham, "Image-guided robotic flexible needle steering," IEEE Trans. Robotics, vol. 23, no. 3, pp. 459-467, Jun. 2007.

[16] M. Horner, L. Ries, M. Krapcho, N. Neyman, R. Aminou, N. Howlader, S. Altekruse, E. Feuer, L. Huang, A. Mariotto, B. Miller, D. Lewis, M. Eisner, D. Stinchcomb, and B. Edwards, "SEER cancer statistics review, 1975-2006, National Cancer Institute. Bethesda, MD," Available: http://seer.cancer.gov/csr/, 2009.

[17] O. Khatib, "Real-time obstacle avoidance for manipulators and mobile robots," Int. J. Robotics Research, vol. 5, no. 1, pp. 90-98, 1986.

[18] D. C. Liu and J. Nocedal, "On the limited memory BFGS method for large scale optimization," Mathematical Programming B, vol. 45, no. 3, pp. 503-528, 1989.

[19] L. A. Lyons, R. J. Webster III, and R. Alterovitz, "Motion planning for active cannulas," in Proc. IEEE/RSJ Int. Conf. on Intelligent Robots and Systems (IROS), Oct. 2009, pp. 801-806.

[20] A. J. Martin, W. A. Hall, C. Roark, P. A. Starr, P. S. Larson, and C. L. Truwit, "Minimally invasive precision brain access using prospective stereotaxy and a trajectory guide." J Magn Reson Imaging, vol. 27, no. 4, pp. 737-743, Apr 2008.

[21] W. S. Newman and N. Hogan, "High speed robot control and obstacle avoidance using dynamic potential functions," in Proc. IEEE Int. Conf. Robotics and Automation (ICRA), Mar. 1987, pp. 14-24.

[22] N. Okazaki, "libLBFGS: a library of Limited-memory Broyden-Fletcher-Goldfarb-Shanno (L-BFGS), J Jan. 2010, http://www. chokkan.org/software/liblbfgs/index.html.

[23] OsiriX, "OsiriX imaging software," Available: http://www.osirixviewer.com, 2009.

[24] W. Park, J. S. Kim, Y. Zhou, N. J. Cowan, A. M. Okamura, and G. S. Chirikjian, "Diffusion-based motion planning for a nonholonomic flexible needle model," in Proc. IEEE Int. Conf. Robotics and Automation (ICRA), Apr. 2005, pp. 4611-4616.

[25] K. B. Reed, V. Kallem, R. Alterovitz, K. Goldberg, A. M. Okamura, and N. J. Cowan, "Integrated planning and image-guided control for planar needle steering," in Proc. IEEE RAS/EMBS Int. Conf. Biomedical Robotics and Biomechatronics (BioRob), Oct. 2008, pp. 819-824.

[26] D. C. Rucker and R. J. Webster III, "Parsimonious evaluation of concentric-tube continuum robot equilibrium conformation," IEEE Trans. Biomedical Engineering, vol. 56, no. 9, pp. 2308-2311, Sep. 2009.

[27] P. Sears and P. Dupont, "A steerable needle technology using curved concentric tubes," in Proc. IEEE/RSJ Int. Conf. on Intelligent Robots and Systems (IROS), Oct. 2006, pp. 2850-2856.

[28] P. Sears and P. E. Dupont, "Inverse kinematics of concentric tube steerable needles," in Proc. IEEE Int. Conf. Robotics and Automation (ICRA), Apr. 2007, pp. 1887-1892.

[29] The International Early Lung Cancer Action Program Investigators, "Survival of patients with stage I lung cancer detected on CT screening," The New England Journal of Medicine, vol. 355, no. 17, pp. 1763-1771, Oct. 2006.

[30] J. Tierny, J. P. Vandeborre, and M. Daoudi, "3D mesh skeleton extraction using topological and geometrical analyses," in 14th Pacific Conference on Computer Graphics and Applications (Pacific Graphics 2006), Taipei, Taiwan, 2006, pp. 85-94.

[31] J. M. Vandeweghe, D. Ferguson, and S. Srinivasa, "Randomized path planning for redundant manipulators without inverse kinematics," in IEEE-RAS Int. Conf. on Humanoid Robots, November 2007.

[32] R. J. Webster III, A. M. Okamura, and N. J. Cowan, "Toward active cannulas: Miniature snake-like surgical robots," in Proc. IEEE/RSJ Int. Conf. on Intelligent Robots and Systems (IROS), 2006, pp. 2857-2863.

[33] R. J. Webster III, J. M. Romano, and N. J. Cowan, "Mechanics of precurved-tube continuum robots," IEEE Trans. Robotics, vol. 25, no. 1, pp. 67-78, Feb. 2009.

[34] J. Xu, V. Duindam, R. Alterovitz, and K. Goldberg, "Motion planning for steerable needles in 3D environments with obstacles using rapidlyexploring random trees and backchaining," in Proc. IEEE Int. Conf. Automation Science and Engineering (CASE), Aug. 2008, pp. 41-46. 